\title{
Breaking classical convexity in Waring's problem: Sums of cubes and quasi-diagonal behaviour
}

\author{
Trevor D. Wooley
}

Mathematics Department, University of Michigan, Ann Arbor, MI, 48109-1003, USA; e-mail: wooley@math.lsa.umich.edu

Oblatum 28-VII-1994 \& 20-IV-1995

\section{Introduction}

The natural interpretation of even moments of exponential sums, in terms of the number of solutions of certain underlying diophantine equations, permits a rich interplay to be developed between simple analytic inequalities, and estimates for those even moments. This interplay is in large part responsible for the remarkable success enjoyed by the Hardy-Littlewood method in its application to numerous problems of additive type. In the absence of such an interpretation, the most effective method for bounding odd and fractional moments, hitherto, has been to apply Hölder's inequality to interpolate linearly between the exponents arising at even moments. The object of this paper is to establish a method for handling all moments of exponential sums over smooth numbers non-trivially, thereby breaking out of the latter (classically) implied convex region of permissible exponents. In view of the great flexibility and applicability of the new iterative methods of Vaughan and Wooley (see, for example, $[13,16,18]$ ), this breakthrough has many consequences. In this paper we confine ourselves to two relatively accessible applications, deriving new bounds for sums of cubes, and strengthening substantially what is known about quasi-diagonal behaviour.

In order to describe the consequences of our new method for mean values of smooth Weyl sums, we shall require some notation. Denote by $\mathscr{A}(P, R)$ the set of $R$-smooth numbers of size at most $P$, that is

$$
\mathscr{A}(P, R)=\{n \in[1, P] \cap \mathbb{Z}: p \mid n \text { and } p \text { prime } \Rightarrow p \leqq R\}
$$

Research supported in part by NSF grant DMS-9303505, an Alfred P. Sloan Research Fellowship and a Fellowship from the David and Lucile Packard Foundation. 
Let $k$ be a fixed positive integer exceeding 2, and define the smooth Weyl sum $f(\alpha)=f(\alpha ; P, R)$ by

$$
f(\alpha ; P, R)=\sum_{x \in \sim /(P, R)} e\left(\alpha x^{k}\right),
$$

where $e(z)$ denotes $e^{2 \pi t z}$. Further, when $s$ is a positive real number, define the mean value $U_{s}(P, R)$ by

$$
U_{s}(P, R)=\int_{0}^{1}|f(\alpha ; P, R)|^{s} d \alpha .
$$

Notice that when $s$ is an even integer, say $s=2 t$, the familiar observation that $|f(\alpha)|^{2}=f(\alpha) f(-\alpha)$, combined with orthogonality, reveals that the integral in (1.3) is equal to the number of solutions of the equation

$$
x_{1}^{k}+\cdots+x_{t}^{k}=y_{1}^{k}+\cdots+y_{i}^{k},
$$

with $x_{i}, y_{i} \in \mathscr{A}(P, R)(1 \leqq i \leqq t)$. Vaughan's seminal work [13] on smooth Weyl sums provides non-trivial exponents, $\lambda_{t}(t \in \mathbb{N})$, with the property that for each $\varepsilon>0$, when $\eta$ is a positive number sufficiently small in terms of $\varepsilon, t$ and $k$, one has

$$
U_{2 t}\left(P, P^{\eta}\right) \ll_{i, t, k} P^{\dot{\lambda}_{t}+i} .
$$

(The significance of such a result should be judged in the light of the well known lower bound card $\left(\mathscr{A}\left(P, P^{\eta}\right)\right) \gg{ }_{\eta} P$.) Moreover, these exponents are substantially smaller than those which follow from corresponding bounds for classical Weyl sums (see [10], Lemma 2.5 and Chapter 5). Although subsequent work of Vaughan and Wooley (see, for example, $[16,18]$ ) improves on these exponents $\hat{\lambda}_{t}$, the theory is limited to handling even moments (that is, integral values of $t$ ). Thus, if $s$ is a positive number, and $t$ is the integer satisfying $2 t \leqq s<2 t+2$, then the best upper bound for the $s$ th moment stemming from the above circle of ideas is that derived from Hölder's inequality, that is

$$
\begin{aligned}
\int_{0}^{1}|f(\alpha ; P, R)|^{s} d \alpha & \leqq\left(\int_{0}^{1}|f(\alpha ; P, R)|^{2 t} d \alpha\right)^{a}\left(\int_{0}^{1}|f(\alpha ; P, R)|^{2 t+2} d \alpha\right)^{b} \\
& \ll P^{a \lambda_{l}+b \lambda_{t+1}+\downarrow},
\end{aligned}
$$

where $a=t+1-s / 2$ and $b=s / 2-t$. We shall refer to a bound arising in this manner as being determined by classical convexity, that is, by interpolating between exponents corresponding to even moments.

It is convenient to describe some notation with which to discuss bounds for the mean values $U_{s}(P, R)$. We shall say that an exponent $\mu_{s}=\mu_{s, k}$ is permissible whenever the exponent has the property that for each $\varepsilon>0$, there exists a positive number $\eta=\eta(\varepsilon, s, k)$ such that whenever $R \leqq P^{\eta}$, one has

$$
U_{s}(P, R) \ll_{i, s, k} P^{\mu_{\zeta, k}+\varepsilon} .
$$

Permissible exponents certainly exist, since for each $s$ the estimate $U_{s}(P, R) \ll P^{s}$ is trivial. 
We are now in a position to state a consequence of our new method which leads to an immediate breaking of classical convexity. In Sect. 4 we establish the following theorem.

Theorem 1.1. Let $k$ be an integer with $k \geqq 3$, and let $s$ and $t$ he real numbers with $s+2 t>4$ and $0<t \leqq 1$. Write $v=s(1-t / 2)^{-1}$. Suppose that $\mu_{s, k}$ and $\mu_{r, k}$ are permissible exponents. Then $\mu_{s+21, k}$ is permissible, where

$$
\mu_{s+2 t}=\mu_{s}(1-\theta)+t+s \theta,
$$

and

$$
0=\frac{(2-t) \mu_{0}-2 \mu_{s}}{(k+1) t+(2-t) \mu_{v}-2 \mu_{s}} .
$$

We note that a result similar to Theorem 1.1, but applicable only when $t=1$ and $s$ is an even integer, has been used in work of Vaughan [15] on Waring's problem.

Corollary. Let $k$ be an integer with $k \geqq 3$. Suppose that $\Delta=\Delta_{k}$ has the property that for each $\&>0$, there exists a positive number $\eta=\eta(\varepsilon, k)$ such that whenever $R \leqq P^{\prime \prime}$, one has

$$
\int_{0}^{1}|f(\alpha ; P, R)|^{6} d \alpha \ll_{i, h} P^{3+\Delta+i} .
$$

Let $\sigma$ be a real number with $0<\sigma \leqq 1$. Then for each $\varepsilon>0$, there exists a positive number $\eta=\eta(\varepsilon, \sigma, k)$ such that whenever $R \leqq P^{\eta}$, one has

$$
\int_{0}^{1}|f(\alpha ; P, R)|^{4+\sigma} d \alpha \ll_{\lfloor, \sigma, k} P^{2+\sigma / 2+\delta(\sigma)+\iota,},
$$

where $\delta(\sigma)=e \Delta \sigma^{\frac{\log (h+1)}{\log 2}}$.

The conclusion of the Corollary is plainly superior to the bound following from classical convexity, which yields a similar result with $\delta(\sigma)=\frac{1}{2} \Delta \sigma$. Although our methods may be used to improve the quality of the bound recorded in the Corollary when $k$ is large, the dependence on $\sigma$, for small $\sigma$, remains the same.

If the breaking of classical convexity were the only consequence of our new method, then one might justifiably describe it as being of somewhat technical interest. However, by recycling the new mean value theorems inside the machinery of the new iterative methods, it is possible to improve on existing bounds for even moments of smooth Weyl sums. Since, in general, a detailed consideration of the optimal permissible exponents deriving from our method entails considerable computational effort, we confine our exposition to the case $k=3$. Thus, in Sect. 5, we establish the following theorem.

Theorem 1.2. Let $\xi$ be the positive root of the polynomial $\xi^{3}+16 \xi^{2}+28 \xi-8$, so that $\xi=0.24956813 \ldots$. Then for each $\varepsilon>0$, there exists a positive 
number $\eta=\eta(8)$ such that whenever $R \leqq P^{\prime \prime}$, one has

$$
\int_{0}^{1}\left|f(\alpha ; P, P)^{2} f(\alpha ; P, R)^{4}\right| d \alpha \ll_{l} P^{3+\xi+\downarrow} .
$$

The estimate (1.7) should be compared with Vaughan [13], Theorem 4.4, where the upper bound

$$
\int_{0}^{1}|f(\alpha ; P, R)|^{6} d \alpha \ll_{i} P^{13 / 4+1}
$$

is established. By considering the underlying diophantine equation one finds that (1.7) is stronger than (1.8). Although our improvement is rather small, it has significant implications for the derivation of lower bounds for $R_{7,3}(n)$, the number of representations of $n$ as the sum of seven positive cubes. It is conjectured that

$$
R_{7,3}(n) \sim \frac{3}{4} \Gamma\left(\frac{4}{3}\right)^{6} \Theta(n) n^{4 / 3},
$$

where $\subseteq(n)$ is the singular series associated with seven cubes,

$$
\Xi(n)=\sum_{q=1}^{\infty} \sum_{\substack{\alpha=1 \\(a, q)=1}}^{q}\left(q^{-1} S(q, a)\right)^{7} e(-a n / q),
$$

and

$$
S(q, a)=\sum_{r=1}^{q} e\left(a x^{3} / q\right) .
$$

But, as noted in the introduction of [14], the inequality (1.8) "combined with a straightforward application of the Hardy-Littlewood method based on Weyl's inequality for cubes fails by an $\varepsilon$ in the exponent" to establish a lower bound for $R_{7,3}(n)$ of the same order of magnitude as (1.9). Indeed, Vaughan [14] was forced to apply a complicated argument, based on an efficient differencing argument restricted to the minor arcs, in order to overcome this difficulty. Theorem 1.2 enables us to give a simple proof of a result slightly stronger than Vaughan's.

Corollary A. Let $n$ be a positive integer, and let $\eta$ denote a positive number. Define $r(n ; \eta)$ to be the number of solutions of the equation

with

$$
x_{1}^{3}+x_{2}^{3}+x_{3}^{3}+y_{1}^{3}+\cdots+y_{4}^{3}=n,
$$

$$
1 \leqq x_{i} \leqq n^{1 / 3} \quad(1 \leqq i \leqq 3) \quad \text { and } \quad y_{i} \in \mathscr{A}\left(n^{1 / 3}, n^{\prime \prime}\right) \quad(1 \leqq j \leqq 4) .
$$

Then when $\eta$ is a sufficiently small positive number, and $\delta$ is sufficiently small,

$$
r(n ; \eta)=\mathscr{C}(\eta) \Theta(n) n^{4 / 3}+O\left(n^{4 / 3}(\log n)^{-\delta}\right),
$$

where $\mathscr{C}(\eta)$ is the positive number defined in equation (5.13) below. 
Although the lower bound for $R_{7,3}(n)$ stemming from (1.11) is likely to be very far from the conjectured asymptotic formula (1.9), in principle one can calculate explicitly a suitable choice for $\eta$, and a value for $\mathscr{C}(\eta)$, in order to provide a comparison.

Let $f(X)$ denote the number of natural numbers not exceeding $X$ which are the sum of three cubes of natural numbers. Also, let $E(X)$ denote the number of natural numbers not exceeding $X$ which are not the sum of four cubes of natural numbers. The problems of providing lower bounds for $f(X)$, and upper bounds for $E(X)$, have long histories (see, for example, $[2,3,4,5,6,7,9,11,12,13])$. Theorem 1.2 permits us to make some further, very small, progress.

Corollary B. With $\xi$ defined in the statement of Theorem 1.2,

$$
f(X) \gg, X^{1-c^{3-1}} \text { and } E(X) \ll, X^{1-\frac{4-6,2}{21}+i} \text {. }
$$

Previously, Vaughan [13] had established that $f^{\prime}(X) \gg_{1} X^{11 / 12-1}$ (note that $1-\xi / 3>0.91681$, whereas $\left.\frac{11}{12}<0.91667\right)$, and Brüdern [2] had established that $E(X) \ll{ }_{i} X^{37 / 42+i}$ (note that $1-(4-6 \xi) / 21<0.88083$, whereas $\frac{37}{42}>$ $0.88095)$. The proofs of the estimates given in Corollary B follow, respectively, a standard application of Cauchy's inequality, and the argument of Brüdern [2]. We therefore give the details of neither proof.

Perhaps the most striking consequences of our new method concern estimates for even moments of smooth Weyl sums, of the form (1.5), when $k$ is large and $t$ is small. We examine the latter situation in Sect. 6, where we are able to show that $\lambda_{s}$ is very close to $s$, a phenomenon we have previously described as quasi-diagonal behaviour (see [19]).

Theorem 1.3. Suppose that $k$ is an integer with $k \geqq 3$, and that $s$ is a real number with $4<s \leqq 4 e^{-1} k^{1 / 2}$. Then the exponent $\mu_{\varsigma_{.} k}$ is permissible, where $\mu_{\uparrow, k}=s / 2+\dot{\delta}_{s, h}$, and

$$
\delta_{s, k}=\frac{8 k^{1 / 2}}{e s} \exp \left(-\frac{16 k}{e^{2} s^{2}}\right) .
$$

With a little effort, one can deduce from work of Vaughan [15] a similar bound with $\dot{\delta}_{s, h}$ bounded roughly in the form $\delta_{s, k} \ll \exp \left(-C\left(\log \left(k / s^{2}\right)\right)^{2}\right)$, for a suitable positive constant $C$ (see also [19], Theorem 2). Theorem 1.3 provides a substantial improvement on the latter bound when $s$ is smaller than about $k^{1 / 2}$.

Let $\mathcal{N}_{h, s}(X)$ denote the number of natural numbers not exceeding $X$ which are the sum of $s$ positive $k$ th powers. It is widely believed that,$N_{k, 3}(X) \asymp X^{3 / k}$ for $k \geqq 3$. We make substantial progress in this direction for large $k$.

Corollary. For each integer $k$ with $k \geqq 3$,

$$
\mathscr{N}_{k, 3}(X) \gg_{k} X^{\frac{3}{h}-e^{-k / 17}}
$$


For comparison Vaughan [13], improving on work of Davenport, had established a similar result with $e^{-k / 17}$ replaced by $k^{-2}$, which he later improved in [15] to an expression of the form $A \exp \left(-C(\log k)^{2}\right)$, for a suitable positive constant $C$. The superiority of our new bound is self-evident.

Our basic method for bounding the mean values $U_{s}(P, R)$ is described in Sects. 2,3 and 4. In common with the iterative methods of Vaughan and Wooley, our strategy is to introduce a strong congruence condition on two of the implicit variables through suitable manipulations. In the former treatments, this process was much simplified by reference to the underlying diophantine equations, a resource unavailable to us here. Thus we are forced to perform delicate rearrangements of exponential sums of a somewhat combinatorial flavour. Oversimplifying the situation considerably, our argument bounds the mean value $U_{s}(P, R)$ in the shape

$$
U_{s}(P, R) \ll\left(P^{\prime \prime} R\right)^{s-3 t}\left(P^{t(1+0)} U_{s-2 t}\left(P^{1-0}, R\right)+T_{s, t}\right),
$$

where $t$ and $\theta$ are real numbers with $0 \leqq 0 \leqq 1 / k$ and $0<t \leqq 1$ to be chosen later, and

$$
T_{s, t}=\int_{0}^{1}\left|F(\alpha)^{t} f\left(\alpha ; P^{1-0}, R\right)^{s-2 t}\right| d \alpha
$$

where

$$
F(\alpha)=\sum_{\substack{u \in \mathcal{\prime}\left\langle\rho^{0} R_{R, R)} \\ u>P^{\prime \prime}\right.}} \sum_{\substack{z_{1}, z_{2} \in \mathcal{A}(P, R) \\ z_{1} \equiv z_{2}\left(\bmod u^{k}\right) \\ z_{1} \neq z_{2}}} e\left(\alpha u^{-k}\left(z_{1}^{k}-z_{2}^{k}\right)\right) .
$$

Thus our argument extracts an efficient difference for arbitrary real $s$, not just for $s$ an even integer. Moreover, and this is crucial for the proof of Theorem 1.3, this efficient differencing may be performed on a fractional number of variables (this is the significance of permitting $t$ to be smaller than 1). The expression $T_{s, t}$ may be estimated in terms of mean values for $F(\alpha)$, and the mean values $U_{w}\left(P^{1-0}, R\right)$ for suitable $w$. Optimising the choices for $\theta$ and $t$ ultimately leads to bounds for the mean values $U_{s}(P, R)$.

In the light of the progress described in this paper, it seems natural to enquire whether similar progress can be made in repeated differencing procedures, paralleling treatments of [16] and [18]. After preliminary investigations of this matter, the author feels confident that such is indeed possible, but only through an argument at least an order of magnitude more complicated than the one described in Sects. 2 and 3. Since the consequences of such a result are not yet clear, and in any case likely to be less accessible than those described above, we have decided to defer any such discussion to a future occasion. We note also that a breaking of classical convexity may also be achieved in the context of Vinogradov's mean value theorem by methods similar to those described here. However, at this stage the author has yet to find an application of such a breakthrough which would justify its exposition. 


\section{The preparation lemma}

In order to ascend to a position from which to bound $U_{s}(P, R)$ in the way alluded to in the introduction, in this section we establish a lemma which prepares to extract an efficient difference within this mean value. We first describe some notation and conventions which will ease the burden of exposition.

Throughout, $k$ will be an integer exceeding 2 , and $s$ will be a positive real number. We use $\varepsilon$ and $\eta$ to denote sufficiently small positive numbers, and $P$ to denote a large positive number depending at most on $k, s, \varepsilon$ and $\eta$. The implicit constants in Vinogradov's well-known notation, $\ll$ and $\gg$, will depend at most on $k, s, \varepsilon$ and $\eta$. Also, we write $[x]$ for the greatest integer not exceeding $x$. We adopt the following convention concerning the numbers $:$ and $R$. Whenever $\varepsilon$ or $R$ appear in a statement, either implicitly or explicitly, we assert that for each $\varepsilon>0$, there exists a positive number $\eta(\varepsilon, s, k)$ such that the statement holds whenever $R \leqq P^{\eta}$. Note that the "value" of $\varepsilon$, and $\eta$, may change from statement to statement, and hence also the dependency of implicit constants on $\varepsilon$ and $\eta$. We observe that since our iterative methods will involve only a finite number of statements (depending at most on $k, s$ and $\varepsilon$ ), there is no danger of losing control of implicit constants through the successive changes in our arguments.

With this notation, an exponent $\mu_{s, k}$ is permissible, in the sense defined in the introduction, provided that

$$
U_{s}(P, R) \ll P^{\mu, k+\varepsilon} .
$$

We record some elementary properties of permissible exponents in the following lemma.

Lemma 2.1. For each positive number $s$, and integer $k$ with $k \geqq 3$, a permissible exponent $\mu_{s, k}$ exists wih $\mu_{s, k} \leqq s$. Moreover, $\mu_{s, k}$ necessarily satisfies the inequality $\mu_{s, k} \geqq s / 2$ when $s \geqq 2$. Further, when $0<s \leqq 4$, one may take $\mu_{s, k}=s / 2$.

Proof. As we noted in the introduction, the estimate $U_{s}(P, R) \ll P^{s}$ is trivial, and hence for each $s$ a permissible exponent $\mu_{s, k}$ exists with $\mu_{s, k} \leqq s$. Next we suppose that $s \geqq 2$, and write $t=[s / 2]$. Then by Hölder's inequality,

$$
\int_{0}^{1}|f(\alpha ; P, R)|^{2 t} d \alpha \ll\left(\int_{0}^{1}|f(\alpha ; P, R)|^{s} d \alpha\right)^{2 / / s} .
$$

Recall the implicit assumption that $R=P^{\eta}$ with $\eta$ a positive number. Then by counting only the diagonal solutions of the diophantine equation underlying the mean value on the left hand side of (2.1), and recalling (1.3), we obtain

$$
U_{s}(P, R) \gg\left(P^{t}\right)^{s / 2 t}=P^{s / 2} .
$$

Consequently, every permissible exponent satisfies $\mu_{s, k} \geqq s / 2$.

Finally we note that the estimate $U_{4}(P, R) \ll P^{2+r}$ is essentially classical, on considering the underlying diophantine equation. Thus an application of 
Hölder's inequality shows that when $0<s \leqq 4$, we have $U_{s}(P, R) \ll P^{\varsigma / 2+1}$. This establishes the final assertion of the lemma.

It is convenient at this point to establish a simple estimate for the mean value $\Psi_{s}(Q ; u, w)$, which we define for positive integers $u$ and $w$ by

$$
\Psi_{s}(Q ; u, w)=\int_{0}^{1}\left|g_{u}\left(\alpha w^{h} ; Q / w\right)^{2} f(\alpha ; Q, R)^{s-2}\right| d \alpha,
$$

where

$$
g_{u}(\alpha ; L)=\sum_{\substack{z \in j / L . R) \\(z, u)=1}} e\left(\alpha z^{k}\right)
$$

Lemma 2.2. Suppose that $s$ is a real number with $s \geqq 2$, that $u, w$ are positive integers, and that $\mu_{s, k}$ is a permissible exponent. Then

$$
\Psi_{s}(Q ; u, w) \ll u^{t} w^{-1} Q^{\mu_{1}+i} .
$$

Proof. In view of the well-known identity involving the Möbius function, we have

$$
g_{u}(\alpha ; Q)=\sum_{z \in \in /(Q, R)} \sum_{c \mid(z, u)} \mu(c) e\left(\alpha z^{k}\right)=\sum_{c \mid u} \mu(c) \sum_{\substack{z \in c(q, R) \\ c \mid z}} e\left(\alpha z^{k}\right) .
$$

Then by Cauchy's inequality, and a standard estimate for the divisor function,

$$
\left|g_{u}(\alpha ; Q)\right|^{2} \ll u^{i} \sum_{c \mid u}\left|f\left(\alpha c^{k} ; Q / c, R\right)\right|^{2}
$$

Consequently,

$$
\Psi_{s}(Q ; u, w) \ll u^{l} \sum_{c \mid u} \int_{0}^{1}\left|f\left(\alpha(c w)^{h} ; Q /(c w), R\right)^{2} f(\alpha ; Q, R)^{s-2}\right| d x .
$$

Then by Hölder's inequality,

$$
\Psi_{s}(Q ; u, w) \ll u^{i} \sum_{c \mid u} \mathscr{I}_{s}(Q ; c w)^{2 / s} \mathscr{I}_{s}(Q ; 1)^{1-2 / s},
$$

where

$$
\mathscr{I}_{s}(Q ; d)=\int_{0}^{1}\left|f\left(\alpha d^{k} ; Q / d, R\right)\right|^{s} d \alpha .
$$

On recalling $(1.3)$ we find that $\mathscr{I}_{s}(Q ; d)=U_{s}(Q / d, R)$. But $\mu_{s, k}$ is permissible, so that by $(2.4)$,

$$
\Psi_{s}(Q ; u, w) \ll u^{i} \sum_{c \mid u} Q^{\mu_{\mathrm{s}}+\iota}(c w)^{-\frac{2}{s}\left(\mu_{s}+i\right)} .
$$

The proof of the lemma is completed on observing that by Lemma 2.1, we have $\mu_{s} \geqq s / 2$.

The preparation lemma makes use of a parameter, $\phi$, associated with the differencing procedure. For each positive number $s$, we take $\phi$ to be a real 
number with $0 \leqq \phi \leqq 1 / k$ to be chosen later. We then take

$$
M=P^{\phi}, \quad H=P M^{-h} \text { and } Q=P M^{-1} .
$$

We shall also make use, in the preparation lemma, of a modified set of smooth numbers, $\mathscr{B}(L, \pi, R)$, defined for prime numbers, $\pi$, by

$$
\mathscr{B}(L, \pi, R)=\{x \in \mathbb{N}: L<x \leqq L \pi, \pi|x, p| x \text { and } p \text { prime } \Rightarrow \pi \leqq p \leqq R\} .
$$

Finally, when $P, M$ and $R$ are real numbers with $P>M$ and $R \geqq 2$, we write

$$
\tilde{f}(\alpha ; P, M, R)=\max _{m>M}\left|\sum_{r \in, \mathcal{H}(P / m, R)} e\left(\alpha x^{h}\right)\right| .
$$

Lemma 2.3. Let $s$ be a real number with $s>4$, and let $t=t(s)$ be a real number with $0<t \leqq 1$. Suppose that $\phi=\phi(s, k)$ satisfies $0<\phi \leqq 1 / k$, and that $D$ is a real number with $1 \leqq D \leqq P^{1 / h}$. Suppose also that $\mu_{\mathrm{s}, h}$ and $\mu_{s-2, h}$ are permissible exponents. Then

$$
U_{s}(P, R) \ll P^{\mu_{1}+\imath} D^{s / 2-\mu_{4}}+M P^{1+\mu_{-}+2+\imath}+P^{\left(\frac{i-1-2 t}{,-2 t} \mu_{\mathrm{s}}+1\right.} V_{\mathrm{s}, t}(P, R),
$$

where

$$
V_{s, t}(P, R)=\sum_{1 \leqq d \leqq D} \sum_{\pi \leqq R} \sum_{u \in \mathscr{R}(M / d, \pi, R)} \sum_{1 \leqq e \leqq Q}(d e)^{-\frac{1-4 t}{2 r-4 t}} \mathscr{I}_{t, u}(d, \pi, e)^{\frac{1}{r-2 t}},
$$

and

$$
\mathscr{J}_{t, u l}(d, \pi, e)=\int_{0}^{1}\left|g_{u}(\alpha ; P /(d e))\right|^{2 t} \tilde{f}\left(\alpha u^{k} ; P /(d e), M / d, \pi\right)^{s-2 t} d \alpha .
$$

Proof. We follow more closely the argument of Vaughan [13], Lemma 2.1 than that of Wooley [18], Lemma 2.2, the former permitting greater control to be exercised when negotiating the many combinatorial difficulties. We begin by writing

$$
|f(\alpha ; P, R)|^{2}=f(\alpha ; P, R) f(-\alpha ; P, R)=\mathscr{F}_{1}(\alpha)+\widetilde{\mathscr{F}}_{2}(\alpha),
$$

where

$$
\mathscr{F}_{1}(\alpha)=\sum_{1 \leqq d \leqq D} \sum_{\substack{1, \in \in,(P, R) \\(1,1)=d l}} e\left(\alpha\left(x^{k}-y^{k}\right)\right)
$$

and

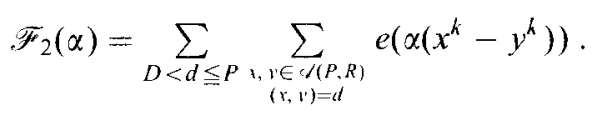

Thus

$$
U_{s}(P, R)=U_{1}+U_{2},
$$


where

$$
U_{i}=\int_{0}^{1} \mathscr{F}_{i}(\alpha)|f(\alpha ; P, R)|^{s-2} d \alpha, \quad(i=1,2) .
$$

We divide into cases. have

(I) Suppose first that $\left|U_{2}\right| \geqq\left|U_{1}\right|$, so that $U_{s}(P, R) \leqq 2\left|U_{2}\right|$. By (2.12) we

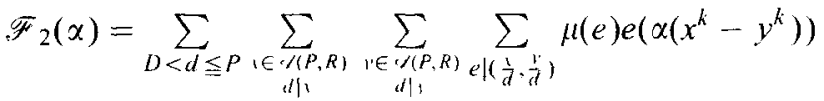

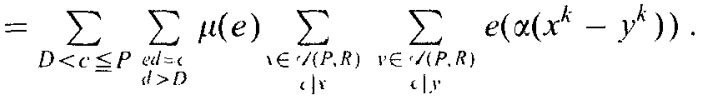

Thus, by using an elementary estimate for the divisor function, we obtain

$$
\mathscr{F}_{2}(\alpha) \ll P^{r:} \sum_{c>D}\left|f\left(\alpha c^{k} ; P / c, R\right)\right|^{2}
$$

Recalling (2.5), and applying Hölder's inequality to (2.13), we deduce that

$$
U_{2} \ll P^{:} \sum_{c>D} \mathscr{I}_{s}(P ; c)^{2 / s} \mathscr{I}_{s}(P ; 1)^{1-2 / s},
$$

and hence obtain

$$
U_{s}(P, R) \ll P^{i}\left(\sum_{c>D}\left(U_{s}(P / c, R)\right)^{2 / s}\right)^{s / 2} .
$$

But $\mu_{s, k}$ is permissible, and thus

$$
U_{s}(P, R) \ll P^{i}\left(\sum_{c>D}(P / c)^{\frac{2}{i}(\mu+\varepsilon)}\right)^{s / 2},
$$

whence the lemma follows in the first case.

(II) Suppose next that $\left|U_{1}\right| \geqq\left|U_{2}\right|$, so that $U_{s}(P, R) \leqq 2\left|U_{1}\right|$. We write

$$
\mathscr{F}_{1}(\alpha)=\mathscr{G}_{1}(\alpha)+\mathscr{G}_{2}(\alpha)
$$

where

$$
\mathscr{G}_{1}(\alpha)=\sum_{1 \leqq d \leqq D} \sum_{\substack{y \in \mathcal{C}(P, R) \\ d \mid y \\ y>M}} \sum_{\substack{x \in \mathcal{G}(P, R) \\(1, y)=d}} e\left(\alpha\left(x^{k}-y^{k}\right)\right)
$$

and

$$
\mathscr{G}_{2}(\alpha)=\sum_{1 \leqq d \leqq D} \sum_{\substack{y \in \alpha(M, R) \\ d \mid y^{\prime}}} \sum_{\substack{r \in \mathcal{d}(P, R) \\(x, y)=d}} e\left(\alpha\left(x^{k}-y^{k}\right)\right)
$$

Thus

$$
U_{s}(P, R) \ll\left|V_{1}\right|+\left|V_{2}\right|
$$


where

$$
V_{i}=\int_{0}^{1} \mathscr{G}_{i}(\alpha)|f(\alpha ; P, R)|^{\beta-2} d \alpha \quad(i=1,2) .
$$

We subdivide into further cases.

(a) Suppose that $\left|V_{2}\right| \geqq\left|V_{1}\right|$, so that $U_{s}(P, R) \ll\left|V_{2}\right|$. By estimating the exponential sum in (2.15) trivially, we find from $(2.16)$ that

$$
V_{2} \ll \sum_{\mathrm{I} \leqq d \leqq D} P M d^{-2} \int_{0}^{1}|f(\alpha ; P, R)|^{s-2} d \alpha \ll P M U_{s-2}(P, R) .
$$

But $\mu_{s-2, h}$ is a permissible exponent, and hence the lemma follows in this case also.

(b) Suppose that $\left|V_{1}\right| \geqq\left|V_{2}\right|$, so that $U_{s}(P, R) \ll\left|V_{1}\right|$. We first observe that from (2.14),

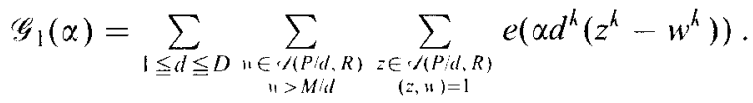

But by Vaughan [13], Lemma 10.1, for each $w$ appearing in the second summation of $(2.17)$, there is a unique triple $(\pi, u, v)$ with $w=u v, \pi$ prime, $u \in \mathscr{B}(M / d, \pi, R)$ and $v \in \mathscr{A}(P /(u d), \pi)$. Thus we deduce that

$$
\mathscr{G}_{1}(\alpha)=\sum_{1 \leqq d \leqq D} \sum_{\substack{\pi \leqq R \\ \pi \text { prme }}} \sum_{u \in \mathscr{B}(M / d, \pi, R)} \mathscr{H}\left(\alpha d^{k}\right),
$$

where

$$
\mathscr{H}(\alpha)=\sum_{n \in \mathcal{d}(P /(u d), \pi)} \sum_{\substack{\in \mathcal{G}(P / d, R) \\(z, u)=1}} e\left(\alpha\left(z^{k}-u^{k} v^{k}\right)\right) .
$$

But

$$
\begin{aligned}
& \mathscr{H}(\alpha)=\sum_{v \in \mathcal{d}(P /(u d), \pi)} \sum_{\substack{z \in d(P i d, R) \\
(z, u)=1}} \sum_{e \mid(z, v)} \mu(e) e\left(\alpha\left(z^{h}-u^{h} v^{h}\right)\right)
\end{aligned}
$$

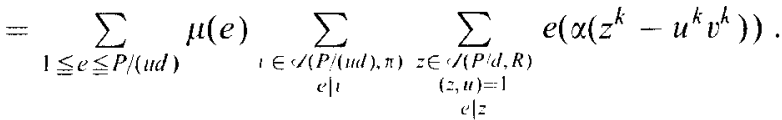

On recalling $(2.8)$, we find that when $u \in \mathscr{B}(M / d, \pi, R)$,

$$
\begin{gathered}
\sum_{\substack{t \in \mathcal{d}(P /(u d), \pi) \\
\ell / \ell}} e\left(\alpha u^{h} v^{h}\right) \ll \max _{m>M / d}\left|\sum_{v \in \mathcal{V}(P /(m d e), \pi)} e\left(\alpha(u e)^{k} v^{h}\right)\right| \\
=\tilde{f}\left(\alpha(u e)^{k} ; P /(d e), M / d, \pi\right) .
\end{gathered}
$$

Then by (2.3) and (2.19),

$$
\mathscr{H}(\alpha) \ll \sum_{1 \leqq e \leqq P /(u d)} \tilde{f}\left(\alpha(u e)^{k} ; P /(d e), M / d, \pi\right)\left|g_{u}\left(\alpha e^{k} ; P /(d e)\right)\right| .
$$


Thus by $(2.16),(2.18)$ and $(2.20)$, we have

$$
V_{1} \ll \sum_{1 \leqq d \leqq D} \sum_{\pi \leqq R} \sum_{u \in S(M / d, \pi, R)} \sum_{1 \leqq e \leqq P / M} W(d, \pi, u, e),
$$

where $W=W(d, \pi, u, e)$ is defined by

$$
W=\int_{0}^{1} \tilde{f}\left(\alpha(u d e)^{h} ; P /(d e), M / d, \pi\right)\left|g_{u}\left(\alpha(d e)^{h} ; P /(d e)\right) f(\alpha ; P, R)^{s-2}\right| d \alpha .
$$

On applying Hölder's inequality to $(2.22)$ we find that

$$
W(d, \pi, u, e) \ll J_{1}^{\frac{1}{,-2 t}} J_{2}^{\frac{1}{2 i-4 t}} J_{3}^{\frac{1-2}{2 i \sim 4 t}}
$$

where

$$
\begin{gathered}
J_{1}=\int_{0}^{1}\left|g_{u}\left(\alpha(d e)^{h} ; P /(d e)\right)\right|^{2 f} \tilde{f}\left(\alpha(u d e)^{h} ; P /(d e), M / d, \pi\right)^{s-2 t} d \alpha, \\
J_{2}=\int_{0}^{1}\left|g_{u}\left(\alpha(d e)^{k} ; P /(d e)\right)^{2} f(\alpha ; P, R)^{s-2}\right| d \alpha
\end{gathered}
$$

and

$$
J_{3}=\int_{0}^{1}|f(\alpha ; P, R)|^{s} d \alpha
$$

Then since $\mu_{s, k}$ is a permissible exponent, we have by (1.3),

$$
J_{3}=U_{s}(P, R) \ll P^{\mu,+t},
$$

and by Lemma 2.2 ,

$$
J_{2}=\Psi_{s}(P ; u, d e) \ll u^{i}(d e)^{-1} P^{\mu_{s}+t} .
$$

Moreover, by a change of variable,

$$
J_{1}=\int_{0}^{1}\left|g_{u}(\alpha ; P /(d e))\right|^{2 t} \tilde{f}\left(\alpha u^{h} ; P /(d e), M / d, \pi\right)^{s-2 t} d \alpha=\mathscr{I}_{1, u}(d, \pi, e)
$$

and consequently the lemma follows in this final case, on combining (2.21) to $(2.26)$.

\section{Efficient differencing}

In order to bound $U_{s}(P, R)$ by using Lemma 2.3 , we must estimate $V_{s, l}(P, R)$. This we do by exploiting an implicit congruence condition between variables occurring inside the mean value $\mathscr{J}_{l, u}$, thereby extracting an efficient difference. When $s$ is even and $t=1$, it is easy to discern the latter congruence condition by considering the underlying diophantine equation. This resource being unavailable to us in general, we make use of an argument somewhat similar to one applied in a minor arc estimate by Vaughan [12], Sect. 8. 
Our proof of Lemma 3.3 below requires bounds for the mean value $\tilde{U}_{s}(P, M, R)$, which we define by

$$
\tilde{U}_{s}(P, M, R)=\int_{0}^{1} \tilde{f}(\alpha ; P, M, R)^{s} d \alpha .
$$

A very convenient approach to providing such bounds is through the use of the Carleson-Hunt theorem (see [8], Theorem 1), which we state below in the form given by Bombieri [1], p12.

Theorem 3.1. Let $T$ be the maximal operator,

$$
T\left(\sum_{n=0}^{N} a_{n} e(n x)\right)=\max _{M \leqq N}\left|\sum_{n=0}^{M} a_{n} e(n x)\right| .
$$

Then $T$ is a bounded operator in $L^{p}$ for $1<p<\infty$. More precisely, there is an absolute constant $B$ such that $\|T\|_{L^{p}} \leqq B p^{5} /(p-1)^{3}$.

We state a corollary of this theorem in the form of a lemma.

Lemma 3.2. Suppose that $s>1$, and that $P, M$ and $R$ are real numbers with $P>M$ and $R>2$. Then

$$
\tilde{U}_{s}(P, M, R) \ll_{s} U_{s}(P / M, R) .
$$

Proof. We apply Theorem 3.1 with

$$
a_{n}= \begin{cases}1, & \text { when } n=x^{k} \text { with } x \in \mathscr{A}(P / M, R), \\ 0, & \text { otherwise, }\end{cases}
$$

and deduce that

$$
\tilde{U}_{s}(P, M, R)=\int_{0}^{1}(T(f(\alpha ; P / M, R)))^{s} d \alpha \ll_{s} \int_{0}^{1}|f(\alpha ; P / M, R)|^{s} d \alpha .
$$

The proof of the lemma is completed on recalling (1.3).

We remark that the deep Carleson-Hunt theorem may be avoided, as pointed out to the author by Professor E. Bombieri, by employing older, less precise, results on the convergence of Fourier series (see, for example, Zygmund [20], Chapter XIII). Indeed, Professor H.L. Montgomery has kindly supplied just such an argument to the author, which we reproduce in the Appendix. The use of the latter argument suffices to establish Lemma 3.2 with the main conclusion replaced by the weaker bound

$$
\tilde{U}_{s}(P, M, R) \ll_{s}(\log P)^{s} U_{s}(P / M, R) .
$$

The latter estimate suffices for our applications. 
Lemma 3.3. Adopt the same notation, and make the same hypotheses, as in the statement of Lemma 2.3. Write, in addition,

$$
\mathcal{U}_{t}=\sum_{1 \leqq d \leqq D} \sum_{\pi \leqq R} \sum_{1 \leqq e \leqq Q} d^{2 t-s / 2} e^{s / 2-1} \cdot \mathscr{T}_{t}(d, \pi, e),
$$

where

$$
\mathscr{T}_{t}(d, \pi, e)=\int_{0}^{1}|\mathscr{K}(\alpha)|^{l} \tilde{f}(\alpha ; P /(d e), M / d, \pi)^{s-2 t} d \alpha,
$$

and

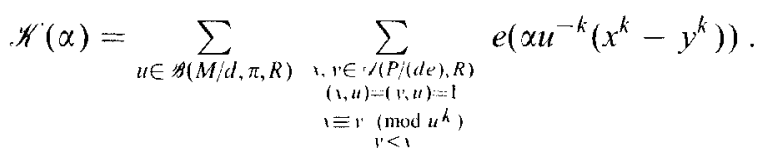

Then if $\mu_{s-2 t, k}$ is a permissible exponent,

$$
V_{s, t}(P, R) \ll P^{t}\left(P^{t} M^{s-2 t} Q^{\mu,-2 t}+M^{s-3 t_{i} \|_{t}}\right)^{\frac{1}{1-2 t}} .
$$

Proof. By applying Hölder's inequality to (2.9), we obtain

$$
V_{s, t}(P, R) \ll \psi_{1}^{\frac{1-1-2 t}{i-2 t}} y_{2}^{\frac{1}{i-2 t}},
$$

where

$$
\mathscr{V}_{i}=\sum_{1 \leqq d \leqq D} \sum_{\pi \leqq R} \sum_{u \in \mathscr{B}(M / d, \pi, R)} \sum_{1 \leqq e \leqq Q} e^{-1},
$$

and

$$
\mathscr{H}_{2}=\sum_{1 \leqq d \leqq D} \sum_{\pi \leqq R} \sum_{u \in \mathscr{B}(M / d, \pi, R)} \sum_{1 \leqq e \leqq Q} d^{2 t-s / 2} e^{s / 2-1} \mathscr{H}_{1, u}(d, \pi, e) .
$$

Moreover a simple estimation yields

$$
\mathscr{V}_{i} \ll P^{i} M R^{2}
$$

In order to estimate $\gamma_{2}$, we make the change of variable $\gamma=\alpha u^{k}$ in (2.10) to obtain

$$
\mathscr{I}_{t, u}=u^{-k} \sum_{l=0}^{u^{k}-1} \int_{l}^{l+1}\left|g_{l t}\left(\gamma u^{-k} ; P /(d e)\right)\right|^{2 t} \tilde{f}(\gamma ; P /(d e), M / d, \pi)^{s-2 t} d \gamma .
$$

Thus, since $\tilde{f}(\gamma ; P /(d e), M / d, \pi)$ is a periodic function of $\gamma$, with period 1 ,

$$
\mathscr{I}_{t, u}=\int_{0}^{1} G_{t, u}(\gamma ; P /(d e)) \tilde{f}(\gamma ; P /(d e), M / d, \pi)^{s-2 t} d \gamma,
$$

where

$$
G_{t, u}(\gamma ; L)=u^{-k} \sum_{l=0}^{u^{k}-1}\left|g_{u}\left((\gamma+l) u^{-k} ; L\right)\right|^{2 t}
$$


It suffices to consider the sum $G_{1, u}(\gamma ; L)$, since by Hölder's inequality,

$$
\sum_{u \in \mathscr{B}(M / d, \pi, R)} G_{l, u}(\gamma ; L) \ll(M R / d)^{1-t}\left(\sum_{u \in \mathscr{B}(M / d, \pi, R)} G_{1, u}(\gamma ; L)\right)^{\prime} .
$$

By orthogonality, it follows from (3.10) that

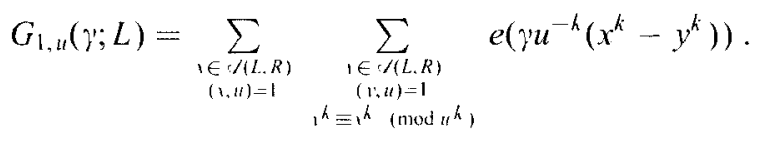

We write

$$
\mathrm{g}(\gamma ; L ; u, z)=\sum_{\substack{1 \in c /(L . R) \\(1, u)=1 \\ 1=z\left(\bmod u^{k}\right)}} e\left(\gamma u^{-k} x^{k}\right),
$$

and let $\mathscr{C}(h, u)$ denote the set of solutions modulo $u^{h}$ to the congruence $x^{h} \equiv h\left(\bmod u^{k}\right)$ with $(x, u)=1$. Then $\operatorname{card}(\mathscr{C}(h, u)) \ll u^{i}$, and so by $(3.12)$, on applying Cauchy's inequality,

$$
G_{1, u}(\gamma ; L)=\sum_{h=1}^{u^{k}}\left|\sum_{z \in \zeta,(h, u)} \mathfrak{g}(\gamma ; L ; u, z)\right|^{2} \leqq u^{\delta} \sum_{h=1}^{u^{k}} \sum_{z \in \zeta,(h, u)}|\mathfrak{g}(\gamma ; L ; u, z)|^{2} .
$$

Thus we arrive at the inequality

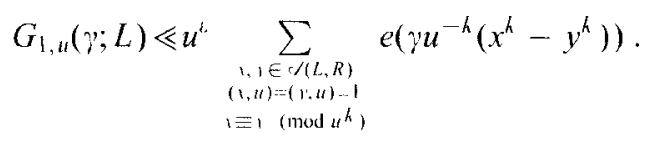

On isolating the diagonal contribution in (3.13), we deduce from (3.11) that

$$
\sum_{u \in \mathscr{B}(M / d, \pi, R)} G_{t, u l}(\gamma ; P /(d e)) \ll P^{i}(M / d)^{1-t}\left(P M\left(d^{2} e\right)^{-1}+|\mathscr{K}(\gamma)|\right)^{t},
$$

where $\mathscr{K}(\gamma)$ is defined by (3.4). Thus, by substituting into (3.7) and (3.9), we obtain

$$
\mathscr{r}_{2} \ll P^{t} M^{1-t}\left(\tilde{T}+\mathscr{U}_{t}\right)
$$

where

$$
\mathscr{T}=(P M)^{t} \sum_{1 \leqq d \leqq D} \sum_{\pi \leqq R} \sum_{1 \leqq e \leqq Q} d^{-s / 2} e^{s / 2-1-1} \int_{0}^{1} \tilde{f}(\gamma ; P /(d e), M / d, \pi)^{s-2 t} d \gamma .
$$

Then by (3.1) and Lemma 3.2, on recalling that $\mu_{s-2 t, k}$ is permissible,

$$
\mathscr{T} \ll(P M)^{i} R \sum_{1 \leqq d \leqq D} \sum_{1 \leqq e \leqq Q} d^{-s / 2} e^{s / 2-1-t}(P /(M e))^{\mu_{1}-2 t+t} .
$$

Furthermore, by Lemma 2.1 we have $\mu_{s-2 t} \geqq s / 2-t$, so that

$$
\mathscr{T} \ll P^{t+i} M^{t} Q^{\mu_{1}-2 t} .
$$


Collecting together (3.6), (3.8), (3.14) and (3.15) completes the proof of the lemma.

The expression $\mathscr{K}(\alpha)$ is somewhat similar to the sum $F_{1}(\alpha)$ appearing in work of Vaughan and Wooley $[13,16,18]$. This observation motivates us to manipulate $\mathscr{U}_{t}$ into a form in which $\mathscr{K}(\alpha)$ can be sanitised inside mean values related to diophantine equations. In the following lemma, we restrict attention to bounds depending only on second and fourth moments of $\mathscr{K}(\alpha)$, our philosophy being that higher moments should be avoided in favour of further efficient differencing operations.

Lemma 3.4. Define the integer $\tau=\tau(k)$ by

$$
\tau(k)=\left\{\begin{array}{l}
1, \quad \text { when } k=3, \text { and when } k \geqq 8 \text { and } k \text { is even }, \\
0, \quad \text { otherwise }
\end{array}\right.
$$

Suppose that $s$ and $t$ are real numbers with $s>4$ and $0<t \leqq 1$. Suppose also that $v$ is a positive number with

$$
\frac{s-2 t}{1-t / 4} \leqq v \leqq \frac{s-2 t}{1-t / 2},
$$

and that $\mu_{n, k}$ is a permissible exponent. Then in the notation of (2.6) and (3.2),

$$
\mathscr{H}_{1} \ll D^{k t} P^{t / 2+i} H^{t-w} M^{t-w+\tau(t / 2-w)} Q^{(1-w) \mu_{2}},
$$

where $w=1-(s-2 t) / v$.

Proof. We apply Hölder's inequality to the integral in (3.3) to obtain

$$
\mathscr{T}_{t}(d, \pi, e) \ll I_{1}^{2 w-1 / 2} I_{2}^{t / 2-w} U_{1}^{1-w},
$$

where

$$
I_{m}=\int_{0}^{1}|\mathscr{K}(\alpha)|^{2 m} d \alpha \quad(m=1,2)
$$

and

$$
U_{i}=\int_{0}^{1} \tilde{f}(\alpha ; P /(d e), M / d, \pi)^{n} d \alpha .
$$

But since $\mu_{v}$ is a permissible exponent, it follows from (3.1) and Lemma 3.2 that

$$
U_{v}=\tilde{U}_{v}(P /(d e), M / d, \pi) \ll U_{v}(P /(M e), \pi) \ll(P /(M e))^{\mu_{t}+t} .
$$

Next, on recalling (3.4), we find that $I_{m}$ is bounded above by the number of solutions $\boldsymbol{u}, \boldsymbol{x}, \boldsymbol{y}$ of the equation

$$
\sum_{i=1}^{m} u_{i}^{-k}\left(x_{i}^{k}-y_{i}^{k}\right)=\sum_{j=m+1}^{2 m} u_{j}^{-k}\left(x_{j}^{k}-y_{j}^{k}\right),
$$


with

$$
\begin{gathered}
M / d<u_{i} \leqq M R / d \quad(1 \leqq i \leqq 2 m) \\
1 \leqq y_{l}<x_{i} \leqq P /(d e) \quad \text { and } x_{l} \equiv y_{l} \quad\left(\bmod u_{l}^{h}\right) \quad(1 \leqq i \leqq 2 m)
\end{gathered}
$$

We now substitute $z_{l}=x_{l}+y_{l}$ and $h_{l}=\left(x_{i}-y_{l}\right) u_{t}^{-k}(1 \leqq i \leqq 2 m)$ into equation (3.20). In view of (3.21) and (3.22), it follows that $1 \leqq h_{i} \leqq(P /(d e))(M / d)^{-h}$ for each $i$. Moreover, we have $2 x_{l}=z_{i}+h_{l} u_{l}^{k}$ and $2 y_{l}=z_{l}-h_{l} u_{l}^{h}(1 \leqq i \leqq 2 m)$. Then on writing

$$
\Psi_{1}(z, h, u)=u^{-h}\left(\left(z+h u^{h}\right)^{h}-\left(z-h u^{h}\right)^{k}\right),
$$

and considering the underlying diophantine equation, it follows that

where

$$
I_{m} \leqq \int_{0}^{1}\left|F_{d, e}(\alpha)\right|^{2 m} d \alpha,
$$

$$
F_{d, e}(\alpha)=\sum_{1 \leqq z \leqq 2 P /(d e)} \sum_{1 \leqq h \leqq H d^{h-1} e^{-1}} \sum_{M / d<u \leqq M R / d} e\left(\alpha \Psi_{1}(z, h, u)\right) .
$$

Thus we may imitate the treatment applied by Vaughan in [13], Sect. 2, and [15], to the case $d=e=1$, and conclude that when $k \geqq 3$,

$$
I_{1} \ll P^{i}(P /(d e))\left(H d^{h-1} e^{-1}\right)(M R / d) \ll P^{1+\varepsilon} H M d^{h-3} e^{-2},
$$

and when $k \geqq 4$,

$$
I_{2} \ll P^{i}(P /(d e))^{2}\left(H d^{k-1} e^{-1}\right)^{3}(M R / d)^{3+\tau} \ll P^{2+t} H^{3} M^{3+\tau} d^{3 h-8-\tau} e^{-5} .
$$

Collecting together (3.2), (3.17), (3.19), (3.25) and (3.26), we find that when $k \geqq 4$,

$$
\mathscr{U}_{t} \ll P^{t / 2+i} H^{t-w} M^{t-w+\tau(t / 2-w)} Q^{\left(1-w^{i}\right) \mu_{l} \cdot U_{t}^{\prime}},
$$

where

$$
\mathscr{U}_{1}^{\prime}=\sum_{1 \leqq d \leqq D} \sum_{\pi \leqq R} \sum_{1 \leqq e \leqq Q} d^{A} e^{-B}
$$

and

$$
A=k t-s / 2, \quad B=(1-w) \mu_{v}+3 t / 2+1-s / 2-w .
$$

But by Lemma 2.1 we have $\mu_{v} \geqq v / 2$, and hence $B \geqq 1+t / 2-w \geqq 1$. Consequently, $\|_{1}^{\prime} \ll P^{t} D^{k t}$, and the lemma follows immediately from (3.27).

When $k=3$ the above argument must be modified, since the fourth moment estimate (3.26) no longer follows from [13] or [15]. We observe instead that by Cauchy's inequality,

$$
\left|F_{1,1}(\alpha)\right|^{2} \leqq H M R \sum_{M<u \leqq M R} \sum_{1 \leqq h \leqq H}\left|\sum_{1 \leqq z \leqq 2 P} e\left(\alpha \Psi_{1}(z, h, u)\right)\right|^{2} \leqq H(M R)^{2} G(\alpha),
$$


where

$$
G(\alpha)=\sum_{1 \leqq h \leqq H} \sum_{1 \leqq z_{1} \leqq 2 P} \sum_{1 \leqq z_{2} \leqq 2 P} e\left(6 \alpha h\left(z_{1}^{2}-z_{2}^{2}\right)\right)
$$

Thus,

$$
\int_{0}^{1}\left|F_{1,1}(\alpha)\right|^{4} d \alpha \leqq H(M R)^{2} \int_{0}^{1} G(\alpha)\left|F_{1,1}(\alpha)\right|^{2} d \alpha .
$$

The integral on the right hand side of (3.28) is equal to the number of solutions of the diophantine equation

$$
6 h\left(z_{1}^{2}-z_{2}^{2}\right)=\Psi_{1}\left(x_{1}, h_{1}, u_{1}\right)-\Psi_{1}\left(x_{2}, h_{2}, u_{2}\right),
$$

with

$$
1 \leqq h, h_{1}, h_{2} \leqq H, \quad M<u_{1}, u_{2} \leqq M R \quad \text { and } \quad 1 \leqq z_{l}, x_{l} \leqq 2 P \quad(i=1,2) \text {. }
$$

If $\Psi_{1}\left(x_{1}, h_{1}, u_{1}\right)=\Psi_{1}\left(x_{2}, h_{2}, u_{2}\right)$ in (3.29), then necessarily $z_{1}=z_{2}$, and so the number of solutions of this type is bounded above by

$$
H P \int_{0}^{1}\left|F_{1,1}(\alpha)\right|^{2} d \alpha \ll P^{2+1} H^{2} M .
$$

On the other hand, if $\Psi_{1}\left(x_{1}, h_{1}, u_{1}\right) \neq \Psi_{1}\left(x_{2}, h_{2}, u_{2}\right)$, then on fixing one of the $O\left((P H M R)^{2}\right)$ possible choices for $x_{l}, h_{l}, u_{i}(i=1,2)$, we find that $h, z_{1}+z_{2}$ and $z_{1}-z_{2}$ are divisors of a fixed non-zero integer. Thus, by using a standard estimate for the divisor function, we find that the number of solutions in this case is bounded above by $P^{\prime}(P H M)^{2}$. It therefore follows from (3.28) that

$$
\int_{0}^{1}\left|F_{1,1}(\alpha)\right|^{4} d \alpha \leqq P^{2+i} H^{3} M^{4},
$$

and the more general estimate (3.26) follows easily. The proof when $k=3$ may therefore be completed as before.

We note that as an alternative to the above strategy, one may cultivate a simple repeated efficient differencing method by applying Hölder's inequality to (3.3). Thus, when $w$ is an integer with $1 \leqq w \leqq(s-2 t) / t$,

$$
\mathscr{T}_{1}(d, \pi, e) \leqq T_{1}^{t / 2} T_{2}^{1-t / 2},
$$

where

$$
\begin{gathered}
T_{1}=\int_{0}^{1}|\mathscr{K}(\alpha)|^{2} \tilde{f}(\alpha ; P /(d e), M / d, \pi)^{2 w} d \alpha, \\
T_{2}=\int_{0}^{1} \tilde{f}(\alpha ; P /(d e), M / d, \pi)^{v} d \alpha,
\end{gathered}
$$

and we write

$$
v=\frac{s-(2+w) t}{1-t / 2} .
$$

On considering the underlying diophantine equation, it is apparent that $T_{1}$ may be estimated as in [18], Sects. 2,3. Meanwhile, by (3.1) and Lemma 3.2, we 
have $T_{2} \ll U_{l}(P /(M e), \pi)$, so that $T_{2}$ may be bounded using ideas within the orbit of this paper.

\section{New permissible exponents}

We now explain how to obtain non-trivial permissible exponents $\mu_{s, h}$ in an iterative manner. Take $\mu_{1, k}(w>0)$ to be known permissible exponents. Initially one might use the trivial exponents $\mu_{11, k}=w$, or exponents obtained from work of Vaughan and Wooley $[13,15,16,18]$ by interpolating between those known for even values of $w$. Let $s$ and $t$ be real numbers with $s>4$ and $0<t \leqq 1$, and suppose that $\phi$ is a real number with $0<\phi \leqq 1 / k$. Let $\gamma$ be a positive number, sufficiently small in terms of $s, t$ and $k$, but large compared to $\varepsilon$, and write $D=P^{i}$. We take $v$ to be a real number with

$$
\frac{s-2 t}{1-t / 4} \leqq v \leqq \frac{s-2 t}{1-t / 2},
$$

and apply Lemmas 3.3 and 3.4 to obtain a bound for $V_{s, t}(P, R)$. Thus, on writing $w=1-(s-2 t) / v$, we obtain

$$
V_{s, t}(P, R) \ll P^{i}\left(M^{s-3 t}\left(\Psi_{1}+D^{k t} \Psi_{2}\right)\right)^{1 /(s-2 t)},
$$

where

$$
\Psi_{1}=(P M)^{t} Q^{\mu_{s}-2 t}
$$

and

$$
\Psi_{2}=P^{t / 2} H^{t-w^{\prime}} M^{t-w+\tau(k)\left(t / 2-w^{\prime}\right)} Q^{\left(1-w^{\prime}\right) \mu_{c}} .
$$

On recalling (2.6), the equation $\Psi_{1}=\Psi_{2}$ implicitly determines a linear equation for $\phi$. Let the solution of this equation be $\theta$, and take

$$
\phi= \begin{cases}0, & \text { when } \theta \leqq 0 \\ 0, & \text { when } 0<0 \leqq 1 / k \\ 1 / k, & \text { when } \theta>1 / k\end{cases}
$$

Then it follows from $(4.1),(4.2),(4.3)$, or from Lemma 3.3, respectively, that

$$
V_{s, \ell}(P, R) \ll P^{i}\left(D^{k t} P^{t} M^{s-2 t} Q^{\mu_{\iota}-2 t}\right)^{1 /(s-2 t)} .
$$

By Lemma 2.3, therefore,

$$
U_{s}(P, R) \ll P^{i}\left(P^{\mu} \cdot D^{s / 2-\mu_{1}}+M P^{1+\mu_{1}-2}+P^{\left(\frac{\imath-1-2 t}{s-2 t}\right) \mu_{s}}\left(D^{k t} P^{\mu_{s}^{\prime}}\right)^{\frac{1}{s-2 t}}\right),
$$

where

$$
\mu_{s}^{\prime}=\mu_{s-2 t}(1-\phi)+t+(s-2 t) \phi .
$$

Observe that when $w>2$, a trivial estimate yields

$$
U_{w}(P, R)=\int_{0}^{1}|f(\alpha ; P, R)|^{w} d \alpha \ll P^{w-2} \int_{0}^{1}|f(\alpha ; P, R)|^{2} d \alpha \ll P^{w-1} .
$$


Consequently, when $s>4$,

$$
\mu_{s}^{\prime}=\mu_{s-2 t}+t+\left(s-2 t-\mu_{s-2 t}\right) \phi \geqq \mu_{s-2 t}+t+\phi .
$$

Furthermore, when $0<t \leqq 1$, an application of Hölder's inequality reveals that $\mu_{s-2} \leqq \frac{s-2}{s-2 t} \mu_{s-2 t}$, and thus by Lemma 2.1 ,

$$
\mu_{s-2 t} \geqq \mu_{s-2}+\frac{2-2 t}{s-2} \mu_{s-2} \geqq \mu_{s-2}+1-t .
$$

Hence by (4.6),

$$
\mu_{s}^{\prime} \geqq \mu_{s-2}+1+\phi
$$

We claim that $\mu_{s}^{\prime}$ is a permissible exponent. If $\mu_{s}^{\prime} \geqq \mu_{s}$, then our claim is trivial, so we assume that $\mu_{s}^{\prime}<\mu_{s}$. We also assume that $\mu_{s}>s / 2$, for otherwise, by Lemma 2.1, we have $\mu_{s}=s / 2$, and thus by (4.6) and Lemma 2.1,

$$
\mu_{s}^{\prime} \geqq \mu_{s-2 t}+t \geqq \frac{1}{2}(s-2 t)+t=\mu_{s},
$$

which contradicts our earlier assumption. Thus, by (4.4) and (4.7),

$$
U_{s}(P, R) \ll P^{i}\left(P^{\mu} \cdot D^{s / 2-\mu_{s}}+P^{\frac{\left(1-1-2 t \mu_{s}+\mu_{1}^{\prime}+2 t_{i}\right.}{\cdots-2 t}}\right) .
$$

We define a sequence of exponents $\left(\mu_{s}^{(r)}\right)_{r=0}^{\infty}$ by $\mu_{s}^{(0)}=\mu_{\text {, }}$, and for $r \geqq 0$ by

$$
\mu_{s}^{(r+1)}=\max \left\{\mu_{s}^{(r)}+\left(\frac{1}{2} s-\mu_{s}^{(r)}\right) \gamma, \frac{(s-1-2 t) \mu_{s}^{(r)}+\mu_{s}^{\prime}+k t \gamma}{s-2 t}\right\} .
$$

By (4.8), for each $r \geqq 0$ we have $U_{s}(P, R) \ll P^{\mu_{s}^{(r)}+r}$, and thus $\mu_{s}^{(r)}(r \geqq 0)$ are permissible exponents. Moreover, in view of our assumptions above, one finds with little difficulty that $\lim _{r \rightarrow \infty} \mu_{s}^{(r)}=\mu_{s}^{\prime}+k t \gamma$, and consequently, when $\tilde{r}$ is sufficiently large in terms of $s, k$ and $\gamma$, we have $\mu_{s}^{(\tilde{r})} \leqq \mu_{s}^{\prime}+2 k t \gamma$. But $\gamma$ is sufficiently small in terms of $s$ and $k$, so that on recalling our notational conventions, we arrive at the conclusion $U_{s}(P, R) \ll P^{u^{\prime}+i}$, which justifies our claim that $\mu_{s}^{\prime}$ is a permissible exponent.

Given a sequence of permissible exponents $\left(\mu_{s}\right)$, we can define a new sequence $\left(\mu_{s}^{\prime}\right)$ by using the above argument. Defining $\mu_{s}^{*}=\min \left\{\mu_{s}, \mu_{s}^{\prime}\right\}$ for each $s$, we obtain a sequence of exponents $\left(\mu_{s}^{*}\right)$ with $\mu_{s}^{*} \leqq \mu_{s}$ for each $s$. It is now apparent that by repeating this process, we obtain a sequence of sequences $\left(\mu_{s}\right)$, convergent to some limit $\left(\tilde{\mu}_{s}\right)$, and that $\tilde{\mu}_{s}$ is a permissible exponent for each $s$.

We illustrate the conclusions of the above argument by proving Theorem 1.1.

The proof of Theorem 1.1. We suppose that $u$ and $t$ are real numbers with $u+2 t>4$ and $0<t \leqq 1$, and write $v=u(1-t / 2)^{-1}$. We suppose also that 
$\mu_{t, h}$ and $\mu_{r, h}$ are permissible exponents. On applying Lemma 3.4 with $s=u+2 t$, we deduce that

$$
\|_{t} \ll P^{l} D^{h_{1}}(P M H)^{1 / 2} Q^{(1-1 / 2) M_{1}} .
$$

Then following the argument above, our choice of $\phi$ is determined from the equation

$$
(P M)^{t} Q^{t_{t t}}=(P M H)^{t / 2} Q^{(1-t / 2) \mu_{t}}
$$

and thus, on recalling (2.6), we put

$$
0=\frac{(2-t) \mu_{r}-2 \mu_{u}}{(k+1) t+(2-t) \mu_{r}-2 \mu_{u}} .
$$

Observe that by convexity, we have $\mu_{r} \geqq(1-t / 2)^{-1} \mu_{t}$, so that $\theta \geqq 0$. Also, if $0>1 / k$ then by the above argument the exponent $\mu_{s}^{*}$ is permissible, where by $(4.5)$,

$$
\mu_{s}^{*}=\mu_{u}+t+\left(u-\mu_{u}\right) / k \leqq \mu_{u}+t+\left(u-\mu_{u}\right) 0 .
$$

In any case, therefore, the exponent $\mu_{s}^{\prime}=\mu_{u l}(1-\theta)+t+u \theta$ is permissible. Theorem 1.1 follows immediately.

The Corollary to Theorem 1.1 provides estimates for $U_{3}(P, R)$ when $s \leqq 5$. When $s \leqq 4$ we have the estimate $U_{s}(P, R) \ll P^{s / 2+i}$, which by Lemma 2.1 is essentially best possible. Thus the Corollary provides estimates for $U_{s}(P, R)$ for the smallest values of $s$ which interest us.

The proof of the Corollary to Theorem 1.1. For each real number $s$, we shall say that the exponent $\delta_{,}=\delta_{s, h}$ is an associated exponent if $\mu_{s, h}=s / 2+\delta_{s, h}$ is permissible. It follows from Lemma 2.1 that if $\dot{\delta}_{s, k}$ is an associated exponent, then necessarily $\delta_{s} \geqq 0$. Furthermore, the associated exponent $\delta_{\mathrm{s}}=s / 2$ is trivial, and one may take $\delta_{s}=0$ when $0<s \leqq 4$.

Suppose that $s$ is a real number with $2<s \leqq 4$, so that $\delta_{s}=0$ is an associated exponent. We apply Theorem 1.1 with $t=1$ to deduce that if $\delta_{2 s}$ is an associated exponent, then so is $\dot{\delta}_{s+2}$, which we define by

$$
\delta_{s+2}=\frac{\frac{1}{2} s \dot{\delta}_{2 s}}{k+1+\delta_{2 s}} .
$$

Suppose that $\mu_{6, k}=3+\Delta$ is a permissible exponent. Then by applying the relation (4.10) iteratively, we deduce the existence of associated exponents $\delta_{4+21-\ell}$ defined for non-negative integers $I$ by $\delta_{6}=\Delta$, and

$$
\delta_{4+2^{1-1}}=\frac{1+2^{-1}}{k+1} \delta_{4+2^{2-1}} \quad(I \geqq 1) .
$$

It follows that for each $I$, we have

$$
\delta_{4+2^{1-1}} \leqq C_{l}(k+1)^{-1} \Delta,
$$


where

$$
C_{l}=\prod_{l=1}^{l}\left(1+2^{-\imath}\right) .
$$

For each real number $\sigma$ with $0<\sigma \leqq 1$ we define $I(\sigma)$ to be the smallest integer satisfying $2^{-l(\sigma)}<\sigma$. Then by convexity it follows that $\mu_{4+\sigma, k}=$ $2+\sigma / 2+\delta(\sigma)$ is permissible, where $\delta(\sigma) \leqq \delta_{4+21-\ell(\sigma)}$. Moreover, by (4.11) and (4.12),

$$
\delta_{4+2^{1-l(\sigma)}} \leqq C_{\infty}(k+1)^{\frac{\log \pi}{\log 2}} \Delta .
$$

The corollary follows on noting that a simple estimation yields $C_{\infty}<e$. (Indeed, a calculation shows that the latter infinite product is approximately $2.38423 \ldots$.)

\section{Sums of cubes}

In this section we prove Theorem 1.2 and the associated Corollary A, which concern sums of cubes. Throughout this section we therefore suppose that $k=3$. The proof of Theorem 1.2 depends on a non-trivial bound for $U_{5}(P, R)$, which we establish in the following lemma.

Lemma 5.1. Suppose that $\mu_{6,3}$ is a permissible exponent, and write $\delta_{6}=$ $\mu_{6,3}-3$. Then the exponent $\mu_{5,3}=\frac{5}{2}+\delta_{5}$ is permissible, where $\delta_{5}=3 \delta_{6} /\left(8+2 \delta_{6}\right)$.

Proof. On applying the argument of the proof of the Corollary to Theorem 1.1 (given in the previous section), the lemma follows immediately from equation (4.10).

Since Vaughan [13], Theorem 4.4 shows that $\mu_{6,3}=13 / 4$ is permissible, as an immediate consequence of Lemma 5.1 we have that $\mu_{5,3}=\frac{5}{2}+\frac{3}{34}$ is a permissible exponent. The latter exponent is superior to that arising from classical convexity, and it is this observation which is crucial to the proof of Theorem 1.2.

Lemma 5.2. Suppose that $\mu_{t, 3}(t=5,6)$ are permissible exponents, and write $\delta_{t}=\mu_{t, 3}-t / 2$. Then the exponent $\mu_{6,3}^{\prime}$ is permissible, with $\mu_{6,3}^{\prime}=3+2 \phi$ and

$$
\phi=\max \left\{\frac{\delta_{6}}{3+\delta_{6}}, \frac{1+2 \delta_{5}+\delta_{6}}{11+2 \delta_{5}+\delta_{6}}\right\} .
$$

Moreover, for each $\varepsilon>0$, there exists a positive number $\eta=\eta(\varepsilon)$ such that whenever $R \leqq P^{\eta}$, one has

$$
\int_{0}^{1}\left|f(\alpha ; P, P)^{2} f(\alpha ; P, R)^{4}\right| d \alpha \ll_{:} P^{\mu_{6,3}^{\prime}+\cdots} .
$$

Proof: We apply the methods of Vaughan and Wooley (noting, in particular, the remark at the end of Wooley [18], Sect. 3). Take $\phi$ to be a real number 
with $0 \leqq \phi \leqq 1 / 7$, and recall the notation defined in (2.6). Write

$$
F(\alpha)=\sum_{1 \leqq z \leqq 2 P} \sum_{1 \leqq h \leqq H} \sum_{M<m \leqq M R} e\left(2 \alpha h\left(3 z^{2}+h^{2} m^{6}\right)\right) .
$$

Further, when $\mathscr{B} \subseteq[0,1)$, define

$$
I(\mathscr{B})=\int_{\mathscr{B}}\left|F(\alpha) f(\alpha ; 2 Q, R)^{4}\right| d \alpha .
$$

Then by [18], Lemma 2.3 and the argument of Lemma 3.1, as in [16], Lemma 2.1 , we have

$$
\int_{0}^{1}\left|f(\alpha ; P, P)^{2} f(\alpha ; P, R)^{4}\right| d \alpha \ll P^{i} M^{3}\left(P M Q^{2}+I([0,1))\right) .
$$

We remark that by considering the underlying diophantine equation, the integral on the left hand side of (5.3) provides an upper bound for $U_{6}(P, R)$, so that the argument which follows will determine a permissible exponent $\mu_{6,3}$.

Let $\mathrm{m}$ denote the set of points $\alpha$ in $[0,1)$ with the property that whenever there exist $a \in \mathbb{Z}$ and $q \in \mathbb{N}$ with $(a, q)=1$ and $|\alpha q-a| \leqq P Q^{-3}$, then $q>P$. Further, let $\mathfrak{M}=[0,1) \backslash \mathfrak{m}$.

Suppose that $x \in \mathfrak{M t}$, and choose $a \in \mathbb{Z}, q \in \mathbb{N}$ so that $(a, q)=1$, $|\alpha q-a| \leqq P Q^{-3}$, and $q \leqq P^{-1} Q^{3}$. Then $q>P$, and hence, as in the argument of the proof of Vaughan [13], Lemma 3.7,

$$
F(\alpha) \ll P^{\prime}(P M)^{1 / 2} H \text {. }
$$

Also, by applying Hölder's inequality to (5.2), we have

$$
I(\mathrm{~m}) \ll J^{1 / 4} U_{5}^{1 / 2} U_{6}^{1 / 4},
$$

where

$$
J=\int_{11}|F(\alpha)|^{4} d \alpha
$$

and

$$
U_{t}=\int_{0}^{1}|f(\alpha ; 2 Q, R)|^{t} d \alpha \quad(t=5,6) .
$$

But by (5.4), and the argument of [13], Lemma 3.7, we have

$$
J \ll P^{1+\varepsilon} M H^{2} \int_{0}^{1}|F(\alpha)|^{2} d \alpha \ll P^{2+\varepsilon} M^{2} H^{3} .
$$

Thus, on substituting into $(5.5)$, and recalling that $\mu_{t}(t=5,6)$ are permissible exponents, we conclude that

$$
I(\mathfrak{m}) \ll P^{c}(P M)^{1 / 2} H^{3 / 4} Q^{\mu_{5} / 2+\mu_{6} / 4} .
$$


In order to estimate $I(\mathfrak{M})$, we observe that $\mathfrak{M}$ is the union over $a \in \mathbb{Z}$ and $q \in \mathbb{N}$ satisfying $(a, q)=1$ and $0 \leqq a \leqq q \leqq P$, of the intervals

$$
\mathfrak{M}(q, a)=\left\{\alpha \in[0,1):|\alpha q-a| \leqq P Q^{-3}\right\}
$$

When $\alpha \in \mathfrak{M}(q, a) \subseteq \mathfrak{M}$, it follows from [13], Lemmata 3.1 and 3.4 that

$$
F(\alpha) \ll P^{\sharp}\left(H_{1}(\alpha ; q, a)+H_{2}(\alpha ; q, a)\right)
$$

where

$$
H_{1}(\alpha ; q, a)=\frac{P H M}{\left(q+Q^{3}|\alpha q-a|\right)^{2 / 3}} \quad \text { and } \quad H_{2}(\alpha ; q, a)=\frac{P H M^{1 / 2}}{\left(q+Q^{3}|\alpha q-a|\right)^{1 / 2}}
$$

Thus by (5.2),

$$
I(\mathfrak{M}) \ll P^{i}\left(K_{1}+K_{2}\right)
$$

where

$$
K_{i}=\sum_{\substack{0 \leqq a \leq q \leq P \\(a, q)=1}} \int_{\mathfrak{M}(q, a)}\left|H_{i}(\alpha ; q, a) f(\alpha ; 2 Q, R)^{4}\right| d \alpha \quad(i=1,2)
$$

Moreover,

$$
\begin{aligned}
\sum_{\substack{0 \leq a \leq q \leqq P \\
(a, q)=1}} \int_{M, k(q, a)}\left|H_{1}(\alpha ; q, a)\right|^{3} d \alpha & \ll \sum_{1 \leqq q \leqq P} q \int_{0}^{\infty} \frac{(P H M)^{3}}{\left(q+Q^{3} q \beta\right)^{2}} d \beta \\
& \ll P^{\prime}(P H M)^{3} Q^{-3}
\end{aligned}
$$

and

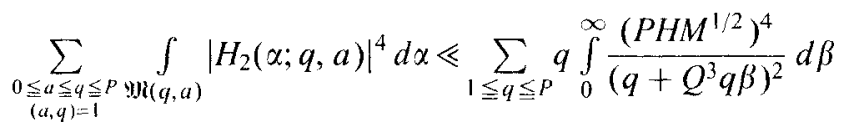

$$
\begin{aligned}
& \ll P^{\prime}(P H)^{4} M^{2} Q^{-3}
\end{aligned}
$$

Consequently, on applying Hölder's inequality to (5.9), we deduce from (5.6) and (5.8) that

$$
I(\mathfrak{M}) \ll P^{1+\varepsilon} H M Q^{-1} U_{6}^{2 / 3}+P^{1+\varepsilon} H M^{1 / 2} Q^{-3 / 4} U_{5}^{1 / 2} U_{6}^{1 / 4}
$$

We now recall (2.6), and deduce from (5.7) and (5.10) that

$$
I([0,1)) \ll P^{\sharp}\left(\Phi_{1}+\Phi_{2}\right),
$$

where

$$
\Phi_{1}=Q^{1+\frac{2}{3} \mu_{6}} \quad \text { and } \quad \Phi_{2}=(P M)^{1 / 2} H^{3 / 4} Q^{\mu_{5} / 2+\mu_{6} / 4} \text {. }
$$

If $\Phi_{1}>\Phi_{2}$, then by $(5.3)$,

$$
\int_{0}^{1}\left|f(\alpha ; P, P)^{2} f(\alpha ; P, R)^{4}\right| d \alpha \ll P^{\varepsilon} M^{3}\left(P M Q^{2}+Q^{1+\frac{2}{3} / \mu_{6}}\right)
$$


whence, on taking $\phi=\delta_{6} /\left(3+\delta_{6}\right)$, we obtain

$$
\int_{0}^{1}\left|f(\alpha ; P, P)^{2} f(\alpha ; P, R)^{4}\right| d \alpha \ll P^{3+2 \phi+i .} .
$$

Meanwhile, if $\Phi_{2}>\Phi_{1}$, then by (5.3),

$$
\int_{0}^{1}\left|f(\alpha ; P, P)^{2} f(\alpha ; P, R)^{4}\right| d \alpha \ll P^{4} M^{3}\left(P M Q^{2}+(P M)^{1 / 2} H^{3 / 4} Q^{\mu_{5} / 2+\mu_{6} / 4}\right) .
$$

In this case we take $\phi=\left(1+2 \delta_{5}+\delta_{6}\right) /\left(11+2 \delta_{5}+\delta_{6}\right)$, and thus once again deduce that the inequality (5.11) holds. Thus on taking $\phi$ as in (5.1), in either case we have the inequality (5.11), and it follows that $\mu_{6,3}^{\prime}=3+2 \phi$ is a permissible exponent. This completes the proof of the lemma.

We now prove Theorem 1.2 by applying Lemmata 5.1 and 5.2 iteratively.

The proof of Theorem 1.2. Suppose that $\mu_{i, 3}(t=5,6)$ are permissible exponents, and write $\mu_{t, 3}=t / 2+\delta_{t}(t=5,6)$. Then by applying Lemmata 5.1 and 5.2 repeatedly, we obtain a sequence of such associated exponents, $\delta_{t}^{(r)}(t=5,6)$, with the property that for each $r$, the exponent $\mu_{t, 3}^{\prime}=t / 2+\delta_{t}^{(r)}(t=5,6)$, is permissible. This sequence is defined by $\delta_{t}^{(0)}=\delta_{t}$, and for $r \geqq 0$ by

$$
\delta_{5}^{(r+1)}=\frac{3 \delta_{6}^{(r)}}{8+2 \delta_{6}^{(r)}} \quad \text { and } \delta_{6}^{(r+1)}=2 \max \left\{\frac{\delta_{6}^{(r)}}{3+\delta_{6}^{(r)}}, \frac{1+2 \delta_{5}^{(r)}+\delta_{6}^{(r)}}{11+2 \delta_{5}^{(r)}+\delta_{6}^{(r)}}\right\} .
$$

On taking the limit as $r \rightarrow \infty$, we thus deduce that the exponent $\mu_{t, 3}^{*}=$ $t / 2+\delta_{t}^{*}(t=5,6)$ is permissible, where $\delta_{5}^{*}$ and $\delta_{6}^{*}$ satisfy the equations

$$
\delta_{5}^{*}=\frac{3 \delta_{6}^{*}}{8+2 \delta_{6}^{*}} \quad \text { and } \quad \delta_{6}^{*}=2 \frac{1+2 \delta_{5}^{*}+\delta_{6}^{*}}{11+2 \delta_{5}^{*}+\delta_{6}^{*}} .
$$

It follows that $\delta_{6}^{*}$ is the positive root of the polynomial $\delta^{3}+16 \delta^{2}+28 \delta-8$, and hence that $\delta_{6}^{*}=0.24956813 \ldots$, and $\delta_{5}^{*}=0.08809182 \ldots$. The proof of Theorem 1.2 is completed on noting that the inequality (1.7) also follows in the above manner, in view of the last line of Lemma 5.2.

Our proof of Corollary A to Theorem 1.2 will be very brief, since with our new permissible exponent $\mu_{6,3}<13 / 4$, an entirely routine argument along the lines of $[13$, Sect. 5] suffices.

The proof of Corollary $A$ to Theorem 1.2. Suppose that $n$ is a large positive integer, and that $\eta$ is a sufficiently small positive number. Let $r(n ; \eta)$ be as in the statement of Corollary A. On writing $P=n^{1 / 3}$ and $R=P^{3 \eta}$, it follows that

$$
r(n ; \eta)=\int_{0}^{1} f(\alpha ; P, P)^{3} f(\alpha ; P, R)^{4} e(-\alpha n) d \alpha .
$$

Let $m$ denote the set of $\alpha \in\left[\frac{1}{6} P^{-2}, 1+\frac{1}{6} P^{-2}\right)$ such that whenever $a \in \mathbb{Z}$ and $q \in \mathbb{N}$ satisfy $(a, q)=1$ and $|\alpha-a / q| \leqq(6 q)^{-1} P^{-2}$, then $q>P$. A standard 
application of Weyl's inequality (see, for example, [10], Lemma 2.4) shows that $\sup _{x \in m}|f(\alpha ; P, P)| \ll P^{3 / 4+\varepsilon}$. Then

$$
\int_{111} f(\alpha ; P, P)^{3} f(\alpha ; P, R)^{4} e(-\alpha n) d \alpha \ll P^{3 / 4+1} \int_{0}^{1}\left|f(\alpha ; P, P)^{2} f(\alpha ; P, R)^{4}\right| d \alpha,
$$

and thus, by the conclusion (1.7) of Theorem 1.2, and in view of the observation that $3+\xi+3 / 4<4$, for some $\delta>0$ we have

$$
\int_{\mathrm{II}} f(\alpha ; P, P)^{3} f(\alpha ; P, R)^{4} e(-\alpha n) d \alpha \ll P^{4-\delta} .
$$

Owing to the presence of three classical Weyl sums, $f(\alpha ; P, P)$, in the expression (5.12), the argument of [13], Sect. 5 suffices to complete the proof of Corollary A, showing that when $\eta$ is sufficiently small, there is a positive number $v$ such that

$$
r(n ; \eta)=\mathscr{C}(\eta) \subseteq(n) n^{4 / 3}+O\left(n^{4 / 3}(\log n)^{-r}\right),
$$

where $\subseteq(n)$ is the singular series defined in the introduction,

$$
\mathscr{C}(\eta)=n^{-4 / 3} \sum_{\mathbf{x}, \mathbf{y}} 3^{-7}\left(x_{1} x_{2} x_{3} y_{1} \ldots y_{4}\right)^{-2 / 3} \rho\left(\frac{\log y_{1}}{3 \eta \log n}\right) \cdots \rho\left(\frac{\log y_{4}}{3 \eta \log n}\right),
$$

and the multiple sum is over $\boldsymbol{x}$ and $\boldsymbol{y}$ with

$$
1 \leqq x_{i} \leqq n \quad(1 \leqq i \leqq 3), \quad n^{3 n}<y_{1} \leqq n \quad(1 \leqq j \leqq 4),
$$

and

$$
x_{1}+x_{2}+x_{3}+y_{1}+\cdots+y_{4}=n \text {. }
$$

Here, $\rho(x)$ denotes Dickman's function, defined for real $x$ by

$$
\begin{aligned}
& \rho(x)=0 \text { when } x<0, \\
& \rho(x)=1 \text { when } 0 \leqq x \leqq 1, \\
& \rho \text { is continuous for } x>0, \\
& \rho \text { is differentiable for } x>1, \\
& x \rho^{\prime}(x)=-\rho(x-1) \text { when } x>1 .
\end{aligned}
$$

\section{Quasi-diagonal behaviour}

The object of this section is to prove Theorem 1.3. Since the proof of the Corollary to Theorem 1.3 follows a well-known argument involving Cauchy's inequality, we shall not give the details here. Recall the notation described in the proof of the Corollary to Theorem 1.1; for each real number $s$, we say that the exponent $\delta_{s}=\delta_{s, k}$ is an associated exponent if $\mu_{s, k}=s / 2+\delta_{s, k}$ is 
permissible. Thus, by Theorem 1.1, for each $t$ with $0<t \leqq 1$, whenever $\delta_{s}$ and $\delta_{s /(1-t / 2)}$ are associated exponents, then so is $\delta_{s+2 t}^{\prime}$, where

$$
\delta_{s+2 t}^{\prime}=\delta_{s}(1-\theta)+\frac{1}{2} s \theta,
$$

and

$$
\theta=\frac{(1-t / 2) \delta_{s /(1-t / 2)}-\delta_{s}}{\frac{1}{2}(k+1) t+(1-t / 2) \dot{\delta}_{s /(1-t / 2)}-\delta_{s}} .
$$

By convexity we have $(1-t / 2) \delta_{s /(1-t / 2)} \geqq \delta_{s}$, and hence

$$
\delta_{s+2 t}^{\prime} \leqq \delta_{s}+\frac{s}{k t} \delta_{s /(1-t / 2)} .
$$

The relation (6.1) permits us to set up an iterative process in the following manner. Suppose that $\delta_{s}(0<s \leqq k)$ are associated exponents. We define a new sequence of associated exponents $\left(\delta_{s, l}^{\prime}\right)$ as follows. When $0<s \leqq 4$, we put $\delta_{s, t}^{\prime}=0$, and for each $s>4$ we define $\delta_{s, t}^{\prime}$ by means of the recurrence relations

$$
\delta_{s+2 t, t}^{\prime}-\delta_{s, t}^{\prime}=\frac{s}{k t} \delta_{s /(1-t / 2)} .
$$

Then by the above argument, the sequence $\left(\delta_{s, t}^{\prime}\right)$ consists of associated exponents. We then optimise with respect to the parameter $t$ subject to $0<t \leqq 1$, and repeat the process.

We claim that for each $r \in \mathbb{N}$, and each positive number $s$, the number $\delta_{s}$ is an associated exponent, where

$$
\delta_{s}=\frac{2}{e^{2}}\left(\frac{e^{2} s^{2}}{16 k}\right)^{r} r !
$$

We prove this claim by induction, beginning with the case $r=1$. Notice that when $t=1$, by making the trivial choice $\phi=1 / k$ in the argument of Sects. 2,3 and 4 , it follows from (4.5) that whenever $\delta_{s}$ is an associated exponent, then so is $\dot{\delta}_{s+2}^{\prime}$, where $\delta_{s+2}^{\prime}=\delta_{s}(1-1 / k)+s /(2 k)$. A simple induction therefore leads to the conclusion that $\delta_{s}=s^{2} /(8 k)$ is an associated exponent for each positive $s$. Thus the inductive hypothesis holds when $r=1$.

Suppose now that the inductive hypothesis holds for some positive integer $r$. For each positive number $s$, and each $t$ with $0<t \leqq 1$, we define $\delta_{s, t}^{\prime}$ to be zero when $0<s \leqq 4$, and when $s+2 t>4$ by

$$
\delta_{s+2 t, t}^{\prime}-\delta_{s, t}^{\prime}=\frac{s}{k t} \cdot \frac{2}{e^{2}}\left(\frac{e^{2} s^{2}}{16 k}\right)^{r}(1-t / 2)^{-2 r} r ! .
$$

Then by (6.2) the numbers $\delta_{s, t}^{\prime}$ are associated exponents. Moreover, it follows from (6.4) that

$$
\delta_{s, t}^{\prime} \leqq \frac{2 r !}{e^{2} t k^{r+1}}\left(\frac{e^{2}}{16}\right)^{r}(1-t / 2)^{-2 r} \sum_{1 \leqq l \leqq[s /(2 t)]}(s-2 l t)^{2 r+1} .
$$


But

$$
\sum_{I \leqq I \leqq[s /(2 t)]}(s-2 l t)^{2 r+1}<(2 t)^{2 r+1} \int_{0}^{s /(2 t)}\left(\frac{s}{2 t}-x\right)^{2 r+1} d x=\frac{s^{2 r+2}}{2 t(2 r+2)} .
$$

Thus

$$
\delta_{s, 1}^{\prime}<\frac{8}{e^{4}}\left(\frac{e^{2} s^{2}}{16 k}\right)^{r+1} \frac{r !}{r+1} \frac{(1-t / 2)^{-2 r}}{t^{2}} .
$$

We take $t=2 /(r+1)$, and observe that $(r /(r+1))^{-2 r}<e^{2}$. Consequently,

$$
\delta_{s, t}^{\prime}<\frac{2}{e^{2}}\left(\frac{e^{2} s^{2}}{16 k}\right)^{r+1}(r+1) !
$$

and by (6.3) the inductive hypothesis follows with $r+1$ in place of $r$.

In order to establish Theorem 1.3, we take $r=\left[16 k /\left(e^{2} s^{2}\right)\right]$ in (6.3). We note that by a simple induction, $r ! \leqq r^{r+1 / 2} e^{1-r}$. Then since

$$
16 k /\left(e^{2} s^{2}\right) \geqq r>16 k /\left(e^{2} s^{2}\right)-1,
$$

we finally obtain

$$
\delta_{s}<2 e^{-2} r^{1 / 2} e^{1-r} \leqq \frac{8 k^{1 / 2}}{e s} e^{-16 k /\left(e^{2} s^{2}\right)} .
$$

On taking $s=6$, we have

$$
\delta_{6} / k \leqq \frac{4}{3 e k^{1 / 2}} e^{-4 k /\left(9 e^{2}\right)}
$$

whence the corollary also follows, with a modicum of computation.

\section{Appendix: maximal partial sums}

In this appendix we reproduce an argument supplied by Professor H.L. Montgomery which establishes a conclusion almost as strong as Lemma 3.2, but which avoids using the difficult Carleson-Hunt Theorem. Before stating this conclusion, we will require some notation. We suppose that $f \in L^{\prime}(\mathbb{T})$, and that $f$ has Fourier coefficients $\hat{f}(k)$. Let $s_{K}(x)$ denote the $K$ th symmetric partial sum of the Fourier series of $f$, so that

$$
s_{K}(x)=\sum_{k=-K}^{K} \hat{f}(k) e(k x)
$$

In addition, put

$$
s_{K}^{*}(x)=\max _{1 \leqq k \leqq K}\left|s_{k}(x)\right|
$$


Finally, we define the $L^{p}$-norm of $f$ in the usual way, so that for $1 \leqq p<\infty$,

$$
\|f\|_{p}=\left(\int_{0}^{1}|f(x)|^{p} d x\right)^{1 / p} .
$$

Lemma 7.1. Suppose that $p$ is a real number with $1 \leqq p \leqq \infty$, and $f \in$ $L^{p}(\mathrm{~T})$. Then for each $K$ with $K \geqq 1$ one has

$$
\left\|s_{K}^{*}\right\|_{p} \leqq\|f\|_{p} \log (6 K) .
$$

Proof. Let $D_{K}(x)$ denote Dirichlet's kernel,

$$
D_{K}(x)=\sum_{h=-K}^{K} e(k x)=\frac{\sin ((2 K+1) \pi x)}{\sin (\pi x)} .
$$

Then

$$
s_{h}(x)=\int_{\mathbb{T}} f(x+y) D_{h}(-y) d y .
$$

Put

$$
D_{K}^{*}(x)=\min \left\{2 K+1,|\sin (\pi x)|^{-1}\right\},
$$

so that $\left|D_{k}(x)\right| \leqq D_{K}^{*}(x)$ for all $k \leqq K$. Then on applying the triangle inequality to equation (7.1), we obtain

$$
s_{K}^{*}(x) \leqq \int_{\mathbb{T}}|f(x+y)| D_{K}^{*}(y) d y .
$$

Suppose first that $1<p<\infty$, and write

$$
I=\int_{\mathbb{T}} s_{K}^{*}(x)^{p} d x
$$

Then by (7.2),

$$
I=\int_{\mathbb{T}} s_{K}^{*}(x)^{p-1} \int_{\mathbb{T}}|f(x+y)| D_{K}^{*}(y) d y d x .
$$

Consequently, on applying Hölder's inequality, we deduce that

$$
I \leqq\left(\iint_{\mathbb{T}^{2}} s_{K}^{*}(x)^{p} D_{K}^{*}(y) d y d x\right)^{1-1 / p}\left(\iint_{\pi^{2}}|f(x+y)|^{p} D_{K}^{*}(y) d y d x\right)^{1 / p}
$$

whence

$$
I \leqq I^{1-1 / p}\|f\|_{p} \int_{\mathbb{T}} D_{K}^{*}(y) d y .
$$

We therefore obtain, for $1<p<\infty$, the inequality

$$
\left\|s_{K}^{*}\right\|_{p} \leqq\|f\|_{p} \int_{\mathbf{I}} D_{K}^{*}(y) d y .
$$

Further, when $p=1$ the inequality (7.3) follows by integrating both sides of (7.2). Moreover (7.3) follows immediately from (7.2) when $p=\infty$. 
Since (7.3) holds for all $p$ with $1 \leqq p \leqq \infty$, in order to complete the proof of the lemma it suffices to show that

$$
\int_{\mathbb{T}} D_{K}^{*}(y) d y<\log (6 K) \text {. }
$$

Since $D_{K}^{*}(y) \leqq 2 K+1$ for $0 \leqq y \leqq(\pi(2 K+1))^{-1}$ and $D_{K}^{*}(y) \leqq(\sin (\pi y))^{-1}$ for $1 /(\pi(2 K+1)) \leqq y \leqq \frac{1}{2}$, it follows that

$$
\begin{aligned}
\int_{\mathbb{T}} D_{K}^{*}(y) d y=2 \int_{0}^{1 / 2} D_{K}^{*}(y) d y & \leqq 2\left(\frac{1}{\pi}+\int_{1 /(\pi(2 K+1))}^{1 / 2} \frac{d y}{\sin (\pi y)}\right) \\
& =\frac{2}{\pi}\left(1+[\log \tan (x / 2)]_{1 /(2 K+1)}^{\pi / 2}\right) \\
& =\frac{2}{\pi}(1+\log \cot (1 /(4 K+2))) \\
& <\frac{2}{\pi} \log (e(4 K+2)) .
\end{aligned}
$$

Since it is simple to verify that this last quantity is at most $\log (6 K)$ for all $K \geqq 1$, the inequality (7.4) follows, and hence the proof of the lemma is complete.

We remark that for $K \geqq 67$, the conclusion of the lemma holds with $\log (6 K)$ replaced by $\log K$.

Acknowledgements. This work was motivated loosely by an idea developed in [17] arising from joint work of the author with Professor R.C. Vaughan. I thank the latter for his many kind words of advice over the years. I also thank Professor E. Bombieri for some advice concerning the Carleson-Hunt theorem, and also for his encouragement. Finally, the author is particularly grateful to Professor H.L. Montgomery for supplying the argument reproduced in the Appendix. The latter provides a relatively simple proof of a conclusion of strength comparable to Lemma 3.2, avoiding the difficult Carleson-Hunt Theorem.

\section{References}

1. E. Bombieri: On Vinogradov's mean value theorem and Weyl sums. Proceedings of the conference on automorphic forms and analytic number theory (Montreal, PQ, 1989), Univ. Montréal, Montreal, PQ, pp. 7-24, 1990

2. J. Brüdern: On Waring's problem for cubes. Math. Proc. Camb. Phil. Soc. 109, 229-256 (1991)

3. H. Davenport: Sur les sommes de puissances entières. C. R. Acad. Sci. 207, 1366-1368 (1938)

4. H. Davenport: On Waring's problem for cubes. Acta Math. 71, 123-143 (1939)

5. H. Davenport: Sums of three positive cubes. J. London Math. Soc. 25, 339-343 (1950)

6. G.H. Hardy, J.E. Littlewood: Some problems of Partitio Numerorum, VI: Further researches in Waring's problem. Math. Z. 23, 1--37 (1925)

7. C. Hooley: On Waring's problem. Acta. Math. 157, 49--97 (1986) 
8. R.A. Hunt: On the convergence of Fourier series, Proceedings of the conference on orthogonal expansions and their continuous analogues (Edwardsville, III. 1967), Southern Illinois Univ. Press, Carbondale, Ill., pp. 235-255, 1968

9. C.J. Ringrose: Sums of three cubes. J. London Math. Soc. (2) 33, 407-413 (1986)

10. R.C. Vaughan: The Hardy-Littlewood Method. University Press, Cambridge, 1981

11. R.C. Vaughan: Sums of three positive cubes. Bull. London Math. Soc. 17, 17-20 (1985)

12. R.C. Vaughan: On Waring's problem for cubes. J. Reine Angew. Math. 365, 122-170 (1986)

13. R.C. Vaughan: A new iterative method in Waring's problem. Acta Math. 162, 1--71 (1989)

14. R.C. Vaughan: On Waring's problem for cubes, II. J. London Math. Soc. (2) 39, 205-218 (1989)

15. R.C. Vaughan: A new iterative method in Waring's problem, II. J. London Math. Soc. (2) 39, 219-230 (1989)

16. R.C. Vaughan, T.D. Wooley: Further improvements in Waring's problem. Acta Math. 174, 147-240 (1995)

17. R.C. Vaughan, T.D. Wooley: Further improvements in Waring's problem, II: Sixth powers. Duke Math. J. 76, 683-710 (1994)

18. T.D. Wooley: Large improvements in Waring's problem. Ann. Math. 135, 131-164 (1992)

19. T.D. Wooley: Quasi-diagonal behaviour in certain mean value theorems of additive number theory, J. Am. Math. Soc. 7, 221-245 (1994)

20. A. Zygmund: Trigonometric series. vol. 2, Cambridge University Press, 1968 